\title{
The Importance of LOX Family Members on Modulating Cell-ECM Interactions in Carcinogenesis
}

\section{Thomas R Cox and Janine T Erler*}

Biotech Research and Innovation Centre (BRIC), University of Copenhagen, Ole Maaløes Vej, 5, Copenhagen 2200, Denmark

\begin{abstract}
The Extracellular Matrix (ECM) presents a fundamentally important local microenvironment for cancer cells in terms of tumour development and progression. A major component of the ECM is the diverse and complex collection of macromolecules, which come together to provide the biochemical and biomechanical cues responsible for controlling individual and collective cell behaviour. Under normal conditions, the tight control of ECM composition and regulation of ECM dynamics (remodelling) is critical to correct organ development and homeostasis.

Dysregulation of these normal ECM dynamics plays an enormous role in diseases such as cancer by disrupting normal cell behaviour. Understanding how changes in ECM remodelling drive cancer is critical to developing efficacious therapies targeting the tumour ECM. In this mini review we focus on the Lysyl Oxidase (LOX) family of proteins and their importance in post-translational modification of ECM components in the context of tumourigenesis.
\end{abstract}

\section{Introduction - Why Cell-ECM Interactions are so Important}

The Extracellular Matrix (ECM) is, in its most basic sense, the "substance between cells". However, such sweeping generalisations undermine the enormously diverse collection of exquisitely finetuned roles the ECM carries out [1]. In fact, the ECM is one of the most important regulators of cellular and tissue function in the body providing as many, if not arguably more, extrinsic cues to a cell than the cell receives intrinsically. Broadly defined, the ECM is the hugely assorted collection of proteins and sugars that surrounds cells in all solid tissues. Although the ECM has historically been perceived as fulfilling a primarily structural and hence biomechanical role, the ability of the ECM to provide the contextual and temporal information responsible for controlling both individual and collective cellular behaviour is becoming increasingly recognised of late. Thus, the ECM plays a significant role in regulating numerous cellular functions, including, but not limited to cell shape, adhesion, migration, proliferation, polarity, differentiation and apoptosis [1]. The precise biochemical constitution of a particular ECM is tissue and organ specific and is, more often than not, critical in defining its function. It does this by providing a gargantuan source of simultaneous biochemical (receptor ligands) and biomechanical (physical) cues which cells integrate to regulate all aspects of cellular behaviour. The differential expression of the 100's of different ECM components currently known to us, is crucial in determining cellular behaviour and phenotype in tissue and organ specific settings $[1,2]$. More importantly however, is the fact that the ECM is by no means a static entity as is highlighted by the fact that it is continuously and progressively remodelled [3]. The tightly controlled, continual remodelling of the ECM is essential, not only for development, and normal organ homeostasis, but also for example during wound healing following injury [4]. Sustained dysregulation often result in life-threatening pathological conditions such as that observed in fibrotic diseases and cancer [2]. This tight regulation is controlled not only at the level of synthesis and secretion, but also post-translationally by large families of proteins that control the stability and turnover of matrix components. In cancer, the aberrant remodelling of the ECM, including increased synthesis, post-translational modification and/or increased breakdown contributes to cancer growth and progression by promoting among other things, cellular transformation, proliferation, angiogenesis, lymphangiogenesis and metastasis $[2,5,6]$. The response of cancer cells to ECM-generated signals and subsequent remodelling is similar to the "dynamic reciprocity" concept proposed by Bisell et al. over 30 years ago [7]. As such, it is thought that normal ECM possesses the ability to provide anti-tumourigenic pressures thus inhibiting cancer progression, and so aberrant changes in the microenvironment will shift the balance of these signals to the pro-cancerous state [8].

In this mini-review, we focus on how ECM remodelling is now thought to be crucial for tumourigenesis and metastatic progression in cancer, focussing on the Lysyl Oxidase (LOX) family of proteins. Whilst there is increasing evidence supporting the intracellular function of several LOX family members and significant progress has been made understanding the intracellular roles of these enzymes, mainly for LOX and LOXL2 [9], reviewed in detail [10,11], this mini-review will focus on extracellular functions which are primarily concerned with post-translational modification of ECM components. Despite these apparently paradoxical intra- and extracellular roles, the LOX family members are rapidly becoming recognised as exciting clinical targets in cancer studies.

\section{The Lysyl Oxidase Family as Regulators of ECM Structure and Stability}

There are five members of the Lysyl Oxidase (LOX) family, all of which are copper dependent amine oxidases. LOX is the classical member of the family with 4 other paralogues; LOX-like 1 (LOXL1), LOX-like 2 (LOXL2), LOX-like 3 (LOXL3) and LOX-like 4 (LOXL4). Each of these members share a high degree of sequence homology within their carboxyl terminal region, with a conserved catalytic domain, *Corresponding author: Janine T. Erler, Biotech Research and Innovation
Centre (BRIC), University of Copenhagen, Ole Maaløes Vej, 5, Copenhagen 2200,
Denmark, E-mail: janine.erler@bric.ku.dk

Received March 05, 2013; Accepted April 20, 2013; Published April 30, 2013

Citation: Cox TR, Erler JT (2013) The Importance of LOX Family Members on Modulating Cell-ECM Interactions in Carcinogenesis. J Carcinogene Mutagene S13: 001. doi:10.4172/2157-2518.S13-001

Copyright: (c) 2013 Cox TR, et al. This is an open-access article distributed unde the terms of the Creative Commons Attribution License, which permits unrestricted use, distribution, and reproduction in any medium, provided the original author and source are credited. 
copper-binding motif, a Lysyl-Tyrosyl-Quinone (LTQ) cofactor and Cytokine Receptor-Like (CRL) domain (Figure 1). The amino termini of the LOX family proteins show a greater degree of diversity and it is thought that this confers their functional specificity. LOX and LOXL1 contain pro-sequences, which are responsible for enabling their secretion as inactive pro-enzymes [12,13], whereas LOXL2, LOXL3 and LOXL4 contain no pro-sequence, but instead contain four scavenger receptor cysteine-rich (SRCR) domains which are thought to be important for function and involved in cell adhesion and proteinprotein interactions in both normal development and cancer $[14,15]$. The primary function of the LOX family is thought to be the covalent crosslinking of collagens and elastin in the Extracellular Matrix (ECM) $[10,16,17]$, which is important for stabilising matrix components and contributing the tensile strength and structural integrity of many tissues. The matrix remodelling functions of LOX and LOXL-2 have been most extensively studied, whilst little is currently known about the remaining family members and is based on conserved sequence homology. In contrast, the expression of the different LOX family members has been more thoroughly studied and has been shown to be tightly controlled during normal development and tissue homeostasis. Aberrant expression and activity of these proteins has been reported in a wide range of diseases linked to abnormal ECM homeostasis, including Scleroderma [18], Menkes' syndrome [19], occipital horn syndrome [20,21], lung fibrosis [14], and liver cirrhosis [22], as well as in many cancers (reviewed in [11]). Of particular interest is evidence showing that LOX family member expression and activity may be co-regulated with ECM components, including those they have been shown to post-translationally modify. This has shown to be either directly or indirectly via growth factor signalling or microRNAs [23]. Such co-regulated ECM components include, but are not limited to tropoelastin [24], fibronectin [25], and collagen I [24,26]. Similarly, the co-regulation with other ECM modifiers such as the Metzincin family of proteins, in particular Matrix Metalloproteinases (MMPs) and their inhibitors Tissue Inhibitor of Metalloproteinase (TIMPs) has also been reported and is discussed in more detail below.

\section{LOX Family Member Mediated ECM Modulation in Primary Tumour Initiation and Progression}

Both the upregulation and downregulation of LOX family members have been reported across different cancer types, a detailed review of which can be found by [11]. In addition to this, the LOX family members have been reported to have both intracellular and extracellular functions suggesting that this family of proteins has complex and paradoxical roles. Indeed they have been described as both tumour suppressors and metastasis promoters which is thought to be linked to their functions both intra- and extracellularly [11]. The extracellular roles of LOX and LOXL2 have been well characterised, especially in diseases such as cancer, yet little is known about the extracellular matrix remodelling function of the other family members in cancer. Nonetheless, all of the family members have been implicated in one way or another with tumourigenesis. In this review, we will focus on the extracellular roles of the LOX family members and in particular LOX and LOXL2, which have primarily been shown to function extracellularly in metastasis promoter roles.

Under normal conditions, the microenvironment has been shown to be capable of suppressing malignant cell growth by providing the correct biochemical (ligand) and biomechanical (physical force) to control normal tissue organisation, cell growth, adhesion and migration. However, tumour-associated ECM has been shown to support malignant cell proliferation, motility and adhesion [27-29]. The underlying mechanisms behind this are slowly being elucidated but there is emerging strong evidence to support the notion that collagen crosslinking by members of the LOX family is crucially important in mediating this malignant transformation process. Data from the Polyoma Middle T (PyMT) and Her2/neu mouse models of breast cancer have shown that the expression of LOX and LOXL2 are critical in driving tumour initiation and metastasis [30,31] as well as in driving tumour cell escape from the primary site, extravasation and growth at secondary sites during metastasis [32,33]. Fibrosis and fibrotic foci are classically associated with tumour progression. Akiri et al. have also that upregulation of LOXL1 in non-invasive breast cancer cells implanted orthotopically in nude mice will act to induce fibrotic foci formation and increase the invasiveness of these cells, indicating that there is an intrinsic connection between tumour fibrosis and tumour invasiveness [34]. Concomitant, increased tissue stiffness resulting from desmoplastic and fibrotic stromal reactions is frequently associated with mammary tumourigenesis and the subsequent progression to metastasis. These reactions have been in

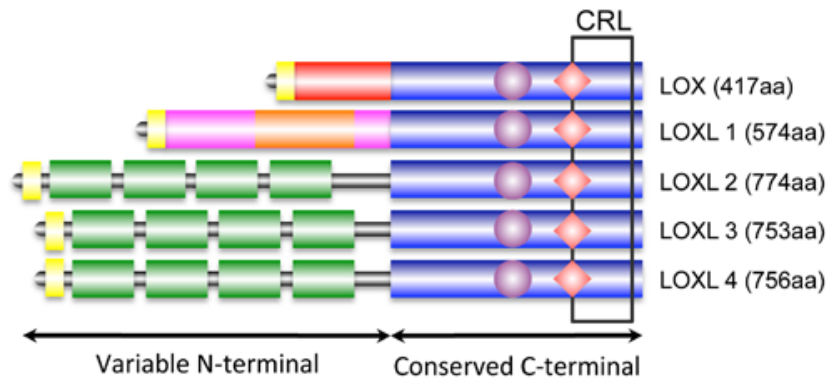

Copper binding domain 
part attributed to LOX-dependent cross-linking of collagen to elastin during neoplastic progression [28,31]. Indeed, Barry-cv has also shown that LOXL2 is also closely associated with desmoplastic stroma [14]. Importantly, the inhibition of secreted LOX family members, and in particular their activity has been shown to reduce not only breast, but also gastric tumour progression in both xenograft and transgenic mouse models [14,31-33,35,36].

Apart from collagen secreted LOX also crosslinks elastin, which contributes to elastin stability [25]. LOX has also been shown to interact with fibronectin, which in turn increases its cross-linking catalytic activity [25] subsequently increasing matrix stiffness. There is also early evidence to suggest that LOX-mediated ECM crosslinking may function in synergy with members of the metzincin superfamily, in particular the matrix metalloproteinases (MMPs), which leads to the remodelling of the ECM in such a way as to increase metastasis. However, although a direct mechanism has not yet been reported, it has been shown that the activation of MMPs is strongly associated with LOX expression in breast cancer [33]. In this paper, the authors postulate that the coordinated action of LOX and MMPs generates a dynamic pro-tumorigenic microenvironment that facts to enhance tumour progression. Furthermore, it has also been shown that secreted LOXL2 is capable of driving breast cancer invasion [14,30], and that LOXL2 is associated with the activation of tissue inhibitor of metalloproteinases 1 (TIMP1) [30,37] as well as MMP9 [14,30]. Such coordinated, dynamic ECM remodelling by opposing matrix modifiers is thought to result in a dynamic increase in integrin activation and resulting subsequent downstream intracellular signalling mechanisms such as Focal Adhesion Kinase (FAK) and MAPK [29].

Concomitantly, in a gastric tumour cell xenograft model, it has been shown by Peng et al., that LOXL2 secreted by the tumour cells can activate SRC-FAK signalling, possibly through increasing local stiffness, although this was never investigated, which results in increased tumour cell invasion and metastasis. However, the authors did not investigate the precise cell surface signal mediators behind these responses [35]. As well as affecting tumour cells directly, LOXL2 has been shown to be up-regulated in tumours leading to increases in ECM cross-linking (in particular collagen IV scaffolding) and stiffening which leads to sprouting angiogenesis and vascularization of the tumour which may be a critical step in driving metastatic dissemination of these tumours [38]. Similarly, we have recently shown in models of colorectal cancer that the activity of secreted LOX is critical to tumourigenesis [39], and that these effects are mediated by both biomechanical changes in matrix stiffness [40] and induction of angiogenesis through stimulation of endothelial cells [41].

The transcriptional regulation of LOX family members, and in particular, the dysregulated expression in certain genetic backgrounds also appears to play an important role in tumourigenesis. Of note is work by Chou and colleagues in which GATA3 has been shown to suppresses metastasis and modulate the tumour microenvironment by regulating microRNA-29b expression which in turn modulates expression of a cluster of ECM genes including lysyl oxidase [23]. Concomitantly Gao et al. have shown that LKB1 inhibits lung cancer progression through lysyl oxidase and extracellular matrix remodelling [42]. The authors showed that aberrant LOX expression in tumour suppressor LKB1 deficient lung cancer acts to promote tumour progression through ECM remodelling. Thus, aberrant LOX expression may play a role in tumourigenesis at multiple levels, although it remains to be seen whether in these cases the aberrant expression of LOX is necessary or sufficient for tumour progression in the given context.

\section{LOX Family Member Mediated ECM Modulation in Metastasis and the Metastatic Microenvironment}

Traditionally, tumour metastasis has been described as the autonomous spread of renegade cells throughout the body, driven by cell intrinsic mediators. However, this fails to explain the complexity of tumour metastasis and it is now becoming clear that the ECM and in particular ECM remodelling is crucial for facilitating both escape from the primary tumour, but also for the successful colonisation of secondary metastatic sites. Recently, an exciting new idea has been put forward, the so-called pre-metastatic niche [43]. This concept proffers that for some solid tumours to successfully colonise a secondary site, they rely in part, on the correct appropriation of local environments through ECM remodelling prior to their arrival. This appropriation results in the creation of these so-coined 'pre-metastatic niches' that are permissive to tumour cell colonisation and outgrowth. The notion that primary tumour cells can drive such appropriation systemically, in advance of their arrival, at distinct and seemingly inhospitable sites with which they have previously had no connection, is an exciting and terrifying concept and highlights the therapeutic potential of targeting ECM remodelling (discussed later) to prevent the metastatic dissemination of solid tumours.

With this in mind, we have recently demonstrated a critical role for tumour-secreted LOX and matrix remodelling in pre-metastatic niche formation [33]. Here we showed that tumour-driven ECM remodelling and matrix stiffening at sites distant from the primary tumour is LOX dependent [44] and acts to recruit bone-marrowderived cells (BMDCs) to these sites [33]. This results in changes in MMP activity and ECM remodelling which acts to facilitate tumour cell colonisation and growth. In support of this, we showed that matrix stiffening either enzymatically (with LOX) or chemically (through glycation) increases invasiveness of the myeloid $(\mathrm{CD} 11 \mathrm{~b}+)$ precursor BMDCs and subsequently increases MMP activity in vitro [33] leading to ECM remodelling. In vivo this manifests as the generation of growth permissive niches capable of supporting enhanced tumour cell colonisation and outgrowth [45]. In support of this work, Wong and colleagues [46] later uncovered a critical role for hypoxia-inducible factor-1 HIF-1 induced expression of LOX family members (LOX, LOXL2 and LOXL4) in the formation of metastatic niches in breast cancer. The authors show that collagen cross-linking in the metastatic lung by these family members led to the recruitment of BMDCs. Whilst, they show that the precise subset of LOX family members expressed varies between individual breast cancers, the expression of HIF-1 was required in each case suggesting that metastatic niche formation is an HIF-1-dependent event during breast cancer progression. Combined with the previously mentioned studies on LOX mediated remodelling in fibrotic environments and at primary tumour sites, data suggests that aberrant LOX-mediated ECM remodelling may be an important mediator of multiple stages of tumourigenesis.

\section{Stromal-Derived LOX Family-Mediated ECM Remodelling in Cancer}

We have already discussed the importance of tumour cell derived LOX-mediated ECM remodelling in tumourigenesis and other disease conditions. Of note is the significant increase in matrix stiffness associated with LOX family ECM remodelling driven by stromal cells. For example, breast cancer tissue is typically stiffer, by up to one order of magnitude, than normal breast tissue $[31,47]$ and it has been shown that this increase is due, in part, to the secretion of LOX and LOXL2 $[31,48]$. Whilst hypoxic tumour cells express high levels of some of the 
LOX family members, in particular LOX, LOXL2 and LOXL4 (reviewed in depth [11]), Levental et al. have shown that stromal derived LOX is one of the key mediators of pre-malignant tissue stiffening which acts to promote tumourigenesis [31]. Whilst aberrant stromal LOX expression is capable of enhancing tumourigenesis, the authors show that it is insufficient to initiate tumourigenesis alone indicating that disruption of normal ECM homeostasis is a co-conspirator rather than a primary inducer of tumourigenesis in models of breast cancer [31]. Concomitant to this, we have shown that in situations of chronic ECM remodelling, such as that found in fibrotic tissue, LOX derived from resident activated fibroblasts in injured tissue is a critical mediator of disease manifestation. The presence of stromal-derived, LOXdependent fibrotic remodelling in organs leads to the generation of a growth permissive milieu capable of enhancing and accelerating metastatic tumour cell colonisation and outgrowth [49]. These LOX-mediated effects lead to increased collagen crosslinking and elevated matrix stiffness, which acts to support disseminated tumour cell colonisation and outgrowth through activation of SRC kinase leading to enhanced proliferation. Thus, in both situations aberrant ECM remodelling, driven in a LOX-dependent manner by stromal cells leads to the generation of growth permissive milieu critical to tumourigenesis.

\section{Targeting LOX Family Mediated ECM Modulation in Cancer}

The therapeutic targeting of extracellular proteins is becoming hugely attractive in light of evidence implicating the tumour microenvironment as pivotal in all aspects of tumour initiation and progression. At present, there is a discrete lack of specific inhibitors for the LOX family members, although there are multiple programs underway to develop novel small molecule inhibitors. In spite of this, and due to the predominantly extracellular function of LOX family members, the use of immunological inhibition has shown great success in vitro and in vivo and also allows a greater degree of specificity and control. Despite the limitations in delivery of antibodybased deliveries, and promiscuity of the small molecule inhibitors, targeting the extracellular roles of the LOX family members makes for a promising approach. In particular, many studies involving albeit non-selective inhibitors of LOX and LOXL2 catalytic activity have demonstrated a marked reduction in metastatic spread [14,30$33,35,49]$. Another interesting avenue is the possibility of targeting the upregulation of LOX family members as an underlying cause of tumour associated fibrosis that is typically observed in more aggressive cancers. For example, as discussed above, the study by Wong et al. has demonstrated that targeting HIF1-alpha, which they show to regulate expression of LOX, LOXL2 and LOXL4 family members, leads to a decrease in LOX family member expression and decrease in the development of tumour associated fibrosis thereby attenuating tumour progression and metastasis [46]. Altogether, emerging data across all laboratories would suggest that targeting the activity of LOX family members may act to reduce the development of fibrotic foci and therefore act to improve prognosis.

Despite the potential promise, targeting a family of proteins with such an array of biological functions (reviewed in depth in [11]), the lack of known crystal structures and little or no reported functional redundancy, is a challenging and exciting prospect. Owing to the complexity of involvement of the LOX family, it is essential to have a solid understanding of the networks being targeted [50-52]. At present there is limited evidence to suggest any functional redundancy between the family members, despite showing high levels of sequence homology.
Furthermore, the lack of complete or partial crystal structures for any of the LOX family members currently precludes classical drug development and screening methodologies, and as mentioned previously, even the small-molecule inhibitors currently available are non-selective. Immunological inhibition does allow a greater degree of specificity, however, this is accompanied by increasingly difficult methods of administration and precludes intracellular targeting of the family, which may however be advantageous according to recent reports [53]

The critical importance of the extracellular function of the LOX family of enzymes combined with their relatively low expression level in normal tissues and the dramatic abrogation of cancer progression upon inhibition, make the LOX family highly attractive drug targets. Despite the potential challenges facing researchers, the development of LOX family inhibitors will hopefully bring more efficacious therapies against primary and metastatic tumours to the clinic in the not-toodistant future.

\section{References}

1. Hynes RO (2009) The extracellular matrix: not just pretty fibrils. Science 326 1216-1219.

2. Cox TR, Erler JT (2011) Remodeling and homeostasis of the extracellula matrix: implications for fibrotic diseases and cancer. Dis Model Mech 4: 165178.

3. Yu H, Mouw JK, Weaver VM (2011) Forcing form and function: biomechanical regulation of tumor evolution. Trends Cell Biol 21: 47-56.

4. Lu P, Takai K, Weaver VM, Werb Z (2011) Extracellular matrix degradation and remodeling in development and disease. Cold Spring Harb Perspect Biol 3.

5. Avraamides CJ, Garmy-Susini B, Varner JA (2008) Integrins in angiogenesis and lymphangiogenesis. Nat Rev Cancer 8: 604-617.

6. Lu P, Weaver VM, Werb Z (2012) The extracellular matrix: a dynamic niche in cancer progression. J Cell Biol 196: 395-406.

7. Bissell MJ, Hall HG, Parry G (1982) How does the extracellular matrix direct gene expression? J Theor Biol 99: 31-68.

8. Bissell MJ, Hines WC (2011) Why don't we get more cancer? A proposed role of the microenvironment in restraining cancer progression. Nat Med 17: 320329

9. Moreno-Bueno G, Salvador F, Martín A, Floristán A, Cuevas EP, et al. (2011) Lysyl oxidase-like 2 (LOXL2), a new regulator of cell polarity required fo metastatic dissemination of basal-like breast carcinomas. EMBO Mol Med 3 : 528-544.

10. Kagan HM, Li W (2003) Lysyl oxidase: properties, specificity, and biological roles inside and outside of the cell. J Cell Biochem 88: 660-672.

11. Barker HE, Cox TR, Erler JT (2012) The rationale for targeting the LOX family in cancer. Nat Rev Cancer 12: 540-552.

12. Lucero HA, Kagan HM (2006) Lysyl oxidase: an oxidative enzyme and effector of cell function. Cell Mol Life Sci 63: 2304-2316.

13. Trackman PC, Bedell-Hogan D, Tang J, Kagan HM (1992) Post-translational glycosylation and proteolytic processing of a lysyl oxidase precursor. J Biol Chem 267: 8666-8671.

14. Barry-Hamilton V, Spangler R, Marshall D, McCauley S, Rodriguez HM, et al. (2010) Allosteric inhibition of lysyl oxidase-like-2 impedes the development of a pathologic microenvironment. Nat Med 16: 1009-1017.

15. Liu X, Zhao Y, Gao J, Pawlyk B, Starcher B, et al. (2004) Elastic fiber homeostasis requires lysyl oxidase-like 1 protein. Nat Genet 36: 178-182.

16. Vadasz Z, Kessler O, Akiri G, Gengrinovitch S, Kagan HM, et al. (2005) Abnormal deposition of collagen around hepatocytes in Wilson's disease is associated with hepatocyte specific expression of lysyl oxidase and lysyl oxidase like protein-2. J Hepatol 43: 499-507.

17. Kim YM, Kim EC, Kim Y (2011) The human lysyl oxidase-like 2 protein functions as an amine oxidase toward collagen and elastin. Mol Biol Rep 38: 145-149. 
Citation: Cox TR, Erler JT (2013) The Importance of LOX Family Members on Modulating Cell-ECM Interactions in Carcinogenesis. J Carcinogene Mutagene S13: 001. doi:10.4172/2157-2518.S13-001

18. Chanoki M, Ishii M, Kobayashi H, Fushida H, Yashiro N, et al. (1995) Increased expression of lysyl oxidase in skin with scleroderma. Br J Dermatol 133: 710715

19. Royce PM, Camakaris J, Danks DM (1980) Reduced lysyl oxidase activity in skin fibroblasts from patients with Menkes' syndrome. Biochem J 192: 579-586.

20. Byers PH, Siegel RC, Holbrook KA, Narayanan AS, Bornstein P, et al. (1980) $X$-linked cutis laxa: defective cross-link formation in collagen due to decreased lysyl oxidase activity. N Engl J Med 303: 61-65.

21. Khakoo A, Thomas R, Trompeter R, Duffy P, Price R, et al. (1997) Congenital cutis laxa and lysyl oxidase deficiency. Clin Genet 51: 109-114.

22. Kagan HM (1994) Lysyl oxidase: mechanism, regulation and relationship to liver fibrosis. Pathol Res Pract 190: 910-919.

23. Chou J, Lin JH, Brenot A, Kim JW, Provot S, et al. (2013) GATA3 suppresses metastasis and modulates the tumour microenvironment by regulating microRNA-29b expression. Nat Cell Biol 15: 201-213.

24. Behmoaras J, Slove S, Seve S, Vranckx R, Sommer P, et al. (2008) Differentia expression of lysyl oxidases LOXL1 and LOX during growth and aging suggests specific roles in elastin and collagen fiber remodeling in rat aorta. Rejuvenation Res 11: 883-889.

25. Fogelgren B, Polgár N, Szauter KM, Ujfaludi Z, Laczkó R, et al. (2005) Cellular fibronectin binds to lysyl oxidase with high affinity and is critical for its proteolytic activation. J Biol Chem 280: 24690-24697.

26. Yamazaki Y, Mikami Y, Yuguchi M, Namba Y, Isokawa K (2012) Development of collagen fibres and lysyl oxidase expression in the presumptive dermis of chick limb bud. Anat Histol Embryol 41: 68-74.

27. Weaver VM, Petersen OW, Wang F, Larabell CA, Briand P, et al. (1997) Reversion of the malignant phenotype of human breast cells in threedimensional culture and in vivo by integrin blocking antibodies. J Cell Biol 137 231-245.

28. Paszek MJ, Zahir N, Johnson KR, Lakins JN, Rozenberg GI, et al. (2005) Tensional homeostasis and the malignant phenotype. Cancer Cell 8: 241-254.

29. Butcher DT, Alliston T, Weaver VM (2009) A tense situation: forcing tumour progression. Nat Rev Cancer 9: 108-122.

30. Barker HE, Chang J, Cox TR, Lang G, Bird D, et al. (2011) LOXL2-mediated matrix remodeling in metastasis and mammary gland involution. Cancer Res 71: 1561-1572.

31. Levental KR, Yu H, Kass L, Lakins JN, Egeblad M, et al. (2009) Matrix crosslinking forces tumor progression by enhancing integrin signaling. Cell 139: 891-906.

32. Erler JT, Bennewith KL, Nicolau M, Dornhöfer N, Kong C, et al. (2006) Lysyl oxidase is essential for hypoxia-induced metastasis. Nature 440: 1222-1226.

33. Erler JT, Bennewith KL, Cox TR, Lang G, Bird D, et al. (2009) Hypoxia-induced lysyl oxidase is a critical mediator of bone marrow cell recruitment to form the premetastatic niche. Cancer Cell 15: 35-44.

34. Akiri G, Sabo E, Dafni H, Vadasz Z, Kartvelishvily Y, et al. (2003) Lysyl oxidaserelated protein-1 promotes tumor fibrosis and tumor progression in vivo. Cancer Res 63: 1657-1666.

35. Peng L, Ran YL, Hu H, Yu L, Liu Q, et al. (2009) Secreted LOXL2 is a nove therapeutic target that promotes gastric cancer metastasis via the Src/FAK pathway. Carcinogenesis 30: 1660-1669.

36. Taylor MA, Amin JD, Kirschmann DA, Schiemann WP (2011) Lysyl oxidase contributes to mechanotransduction-mediated regulation of transforming growth factor-Î² signaling in breast cancer cells. Neoplasia 13: 406-418.
37. Offenberg H, Brünner N, Mansilla F, Orntoft Torben F, Birkenkamp-Demtroder $\mathrm{K}$ (2008) TIMP-1 expression in human colorectal cancer is associated with TGF-B1, LOXL2, INHBA1, TNF-AIP6 and TIMP-2 transcript profiles. Mol Onco 2: $233-240$.

38. Bignon M, Pichol-Thievend C, Hardouin J, Malbouyres M, Bréchot N, et al. (2011) Lysyl oxidase-like protein-2 regulates sprouting angiogenesis and type IV collagen assembly in the endothelial basement membrane. Blood 118 3979-3989.

39. Baker AM, Cox TR, Bird D, Lang G, Murray GI, et al. (2011) The role of lysyl oxidase in SRC-dependent proliferation and metastasis of colorectal cancer. $J$ Natl Cancer Inst 103: 407-424.

40. Baker AM, Bird D, Lang G, Cox TR, Erler JT (2013) Lysyl oxidase enzymatic function increases stiffness to drive colorectal cancer progression through FAK Oncogene 32: 1863-1868.

41. Baker AM, Bird D, Welti JC, Gourlaouen M, Lang G, et al. (2013) Lysyl oxidase plays a critical role in endothelial cell stimulation to drive tumor angiogenesis. Cancer Res 73: 583-594.

42. Gao Y, Xiao Q, Ma H, Li L, Liu J, et al. (2010) LKB1 inhibits lung cance progression through lysyl oxidase and extracellular matrix remodeling. Proc Natl Acad Sci U S A 107: 18892-18897.

43. Kaplan RN, Riba RD, Zacharoulis S, Bramley AH, Vincent L, et al. (2005) VEGFR1-positive haematopoietic bone marrow progenitors initiate the premetastatic niche. Nature 438: 820-827.

44. Graham HK, Hodson NW, Hoyland JA, Millward-Sadler SJ, Garrod D et al. (2010) Tissue section AFM: In situ ultrastructural imaging of native biomolecules. Matrix Biol 29: 254-260.

45. Cox TR, Gartland A, Erler JT (2012) The pre-metastatic niche: is metastasis random? BoneKEy Rep 1.

46. Wong CC, Gilkes DM, Zhang H, Chen J, Wei H, et al. (2011) Hypoxia-inducible factor 1 is a master regulator of breast cancer metastatic niche formation. Proc Natl Acad Sci U S A 108: 16369-16374.

47. Lopez JI, Kang I, You WK, McDonald DM, Weaver VM (2011) In situ force mapping of mammary gland transformation. Integr Biol (Camb) 3: 910-921.

48. Ng MR, Brugge JS (2009) A stiff blow from the stroma: collagen crosslinking drives tumor progression. Cancer Cell 16: 455-457.

49. Cox TR, Bird D, Baker AM, Barker HE, Ho MW, et al. (2013) LOX-mediated collagen crosslinking is responsible for fibrosis-enhanced metastasis. Cance Res 73: 1721-1732.

50. Erler JT, Linding R (2010) Network-based drugs and biomarkers. J Pathol 220 : 290-296.

51. Erler JT, Linding R (2012) Network medicine strikes a blow against breas cancer. Cell 149: 731-733.

52. Cox TR, Erler JT (2012) Network biology and the 3-Dimensiona tumor microenvironment: personalizing medicine for the future. Tumor Microenvironment and Therapy 1: 14-18.

53. Herranz N, Dave N, Millanes-Romero A, Morey L, Díaz VM, et al. (2012) Lysy oxidase-like 2 deaminates lysine 4 in histone H3. Mol Cell 46: 369-376.

This article was originally published in a special issue, Cell-Extracellular Matrix Interactions in Carcinogenesis handled by Editor(s). Dr. Claudio Luparello, University of Palermo, Italy 